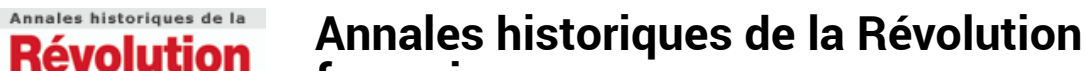

française française

340 | avril-juin 2005

Les arts et la révolution

\section{Post-scriptum : quinze ans après}

\section{Barthélémy Jobert}

\section{OpenEdition}

\section{Journals}

\section{Édition électronique}

URL : https://journals.openedition.org/ahrf/2095

DOI : 10.4000/ahrf.2095

ISSN : 1952-403X

Éditeur :

Armand Colin, Société des études robespierristes

Édition imprimée

Date de publication : 1 juin 2005

Pagination : 105-121

ISSN : 0003-4436

\section{Référence électronique}

Barthélémy Jobert, "Post-scriptum : quinze ans après », Annales historiques de la Révolution française [En ligne], 340 | avril-juin 2005, mis en ligne le 24 avril 2006, consulté le 22 avril 2022. URL : http:// journals.openedition.org/ahrf/2095; DOI : https://doi.org/10.4000/ahrf.2095

Ce document a été généré automatiquement le 22 avril 2022.

Tous droits réservés 


\title{
Post-scriptum : quinze ans après
}

\author{
Barthélémy Jobert
}

1 Il peut paraitre présomptueux de se lancer, en quelques pages, dans un bilan historiographique de l'histoire de l'art français sous la Révolution et l'Empire. C'est pourtant ce qui va être tenté ici, dans un post-scriptum à ce numéro spécial des Annales historiques de la Révolution française, doublement spécial puisque le comité avait voulu ouvrir la revue à des historiens d'art et non à des historiens travaillant sur l'art 1 , distinction significative, et pas seulement en France. Il est néanmoins évident qu'il faut en indiquer les bornes, et en indiquer l'esprit : un bilan historiographique, c'est-à-dire non pas un résumé ou une discussion en profondeur de tel ou tel ouvrage important, mais les grandes directions dans lesquelles s'est orientée, depuis 1989, l'histoire de l'art de cette période; je me limiterai donc aux livres et catalogues d'expositions, n'alourdissant pas un exposé déjà copieux par les références d'articles qui, quand ils sont vraiment majeurs, se retrouvent quasiment toujours en recueil. Il y sera par ailleurs uniquement question des domaines abordés dans ce numéro (ce qui exclut l'architecture, mais inclut la sculpture, un article sur ce sujet précis ayant été primitivement prévu), et des domaines dont je me sens peu ou prou spécialiste, ce qui fait que je n'aborderai pas l'histoire de la musique. Je l'oriente enfin délibérément vers le non-spécialiste : les historiens d'art savent où trouver les recensions complètes des ouvrages dont il sera question ici, alors qu'il n'est pas sûr que l'historien soit toujours au fait du détail de toutes les parutions et de toutes les recherches.

2 L'histoire de l'art occupait une place restreinte, mais honorable, dans les nombreuses publications scientifiques suscitées par le bicentenaire de la Révolution, place originale en même temps car plus peut-être que les historiens pour lesquels la Révolution constitue un objet d'étude en soi, les historiens d'art intègrent l'art des années 1789-1799 dans une chronologie plus large. Il faut ainsi tenir compte, pour les ouvrages parus autour de 1989, du regain antérieur des études sur l'art néo-classique, la traduction en langue française, plus de vingt ans après sa parution en anglais, de l'ouvrage classique de Robert Rosenblum, Transformations in Late Eighteenth Century Art, étant de ce point de vue particulièrement significative ${ }^{2}$. Dans le mouvement plus général du renouveau d'intérêt pour l'art de la seconde moitié du dix-huitième et des premières décennies $\mathrm{du}$ dix-neuvième siècles provoqué par la considérable 
rétrospective organisée à Londres en 1972, The Age of Neo-Classicism ${ }^{3}$, l'exposition du Grand Palais, à Paris, De David à Delacroix. La peinture française de 1774 à $1830^{4}$, complétée par une exposition de dessins ${ }^{5}$, en même temps qu'elle faisait redécouvrir la variété et la richesse de la peinture française de Louis XVI à la Restauration, remettant au jour de nombreux peintres et tableaux tombés dans l'oubli, en fixa, et pour longtemps, un cadre d'analyse d'ensemble: chronologiquement et commodémment fondée sur la succession des régimes politiques, donnant la priorité aux œuvres présentées aux Salons et autres expositions publiques, imposant comme principal axe d'étude la monographie d'artiste, laissant de côté, enfin, une perspective plus européenne (on soulignera néanmoins, au même moment, dans les années 1970-1981, une suite d'expositions introduisant le public parisien aux diverses autres écoles de peinture européennes de cette époque, mais elles aussi envisagées isolément). La commémoration du bicentenaire s'inscrit, historiographiquement, dans cet élan. On en retiendra quelques publications marquantes, à commencer par la remarquable synthèse dirigée par Philippe Bordes et Régis Michel, Aux Armes et aux Arts! Les Arts de la Révolution, 1789-1799, synthèse qui n'a pas été refaite depuis et doit être considérée, à son tour, comme un point de départ ${ }^{6}$. Ouvrage collectif, dont il faut souligner d'emblée qu'il comblait un vide majeur, puisque le seul équivalent qu'on pouvait lui trouver (rendant d'ailleurs encore aujourd'hui bien des services), celui de F. Benoit, datait de près d'un siècle, mais qu'il se voulait aussi, le point est à souligner, critique dans sa démarche. D'abord en envisageant, volontairement, la plupart des « beaux-arts » et pas seulement la peinture, à l'analyse de laquelle se réduit trop souvent l'histoire de l'art. Certes la sculpture et surtout l'estampe sont pratiquement absentes d'Aux Armes et aux Arts !. Mais on y trouve l'architecture et les arts décoratifs, ainsi que des études sur les institutions artistiques ou le discours esthétique et critique. D'autre part, on note, chez presque tous les auteurs, une forte volonté de problématisation, liant, notamment, le politique et l'artistique: démarche qui, si elle est plus courante aujourd'hui, était encore exceptionnelle dans l'histoire de l'art française il y a une quinzaine d'années. Plus encyclopédique, moins maitrisé intellectuellement, le catalogue de l'exposition organisée sous les auspices du Conseil de l'Europe au Grand Palais, La Révolution française et l'Europe, 1789-1799 7, n'a pas eu le même impact chez les historiens d'art. Il en va tout différemment des publications suscitées par la rétrospective David au Louvre, à l'automne 1989, en particulier le catalogue de l'exposition, dû essentiellement à Antoine Schnapper, et les actes du colloque qui lui avait été associé, organisé par Régis Michel. Il est frappant, a posteriori, de constater qu'étaient en grande partie présentes, dès 1989, dans ces divers ouvrages, les grandes directions historiographiques développées depuis.

3 L'art français de la période 1789-1799 n'a pas été négligé, en tant que tel, depuis le Bicentenaire. Il a bénéficié en effet de l'ouverture d'une institution qui lui est plus spécifiquement consacrée, le Musée de la Révolution française installé au Château de Vizille, haut lieu des débuts de la Révolution qui lui a été naturellement affecté, et dont le premier conservateur fut Philippe Bordes, auquel a succédé Alain Chevalier. Ouvert en 1983, il a occupé d'emblée une place importante pour qui s'intéresse aussi bien à la problématique des relations entretenues par la Révolution et la création artistique sous tous ses aspects, et ce, plus encore après 1989 quand, les commémorations du Bicentenaire terminées, le rythme des expositions et des publications sur le sujet s'est considérablement ralenti. Constamment enrichi par des acquisitions qui, c'est à souligner, ne se limitent pas aux seules œuvres d'art françaises, le musée poursuit ainsi 
avec constance une politique d'expositions, de rencontres, de colloques et de publications bien connue de tous les spécialistes de la période. Retenons, plus spécifiquement pour l'histoire de l'art, le catalogue des collections de peintures, de sculptures et de dessins ${ }^{8}$, mais aussi les catalogues d'expositions marquantes, ainsi celle sur la figure de Brutus ${ }^{\text {, }}$ occasionnée par l'acquisition de La Mort de Brutus de Pierre-Narcisse Guérin, ou, tout récemment, celle sur sur le portrait de Lally-Tollendal envoyé par Jean-Baptiste Robin au Salon de 1787, refusé pour des motifs politiques par l'administration royale, mais exposé deux ans plus tard ${ }^{10}$. On doit également au musée l'une des très rares publications touchant à l'histoire de l'estampe sous la Révolution, domaine malheureusement encore trop peu systématiquement exploré en dehors de la seule iconographie ${ }^{11}$.

4 Le musée de la Révolution française constitue néanmoins une exception, dans son approche strictement centrée sur la seule période révolutionnaire. C'est désormais dans un cadre chronologique plus vaste qu'on aborde l'art de la période révolutionnaire, qu'on remonte en amont dans le temps, ou qu'on aille au contraire plus loin, les dernières années du dix-huitième siècle jouant quoi qu'il en soit un rôle charnière, puisqu'elles peuvent tout aussi bien être replacées dans la continuité des Lumières ou du Néo-Classicisme, ou au contraire être prises comme le point de départ d'un art moderne, en rupture, celui du dix-neuvième siècle symbolisé par le Romantisme. De nombreuses synthèses parues dans les années 1990 ont ainsi comme point commun d'avoir retenu une périodisation volontairement très large, où s'inscrit la période révolutionnaire, quel qu'ait été par ailleurs leur point de vue méthodologique, un autre aspect important ayant été de rompre avec une perspective trop francocentrique et d'étendre ainsi l'analyse à l'ensemble de l'Europe. Ainsi de William Vaughan ${ }^{12}$ ou de Werner Hoffman ${ }^{13}$. Il s'agit là de deux spécialistes, qui, audelà des contraintes imposées par les collections où paraissaient leurs ouvrages (plus encyclopédique pour le premier, privilégiant la problématisation des œuvres les plus importantes pour le second), synthétisent ici leurs recherches antérieures. La personnalité et les intérêts de $\mathrm{W}$. Vaughan, spécialiste du Romantisme et de l'art britannique, transparaît dans une vision originale bâtie en grande partie sur une opposition entre le courant classique et le courant romantique, la France ne se joignant que tardivement à ce dernier. Werner Hofmann avait, en tant que directeur de la Kunsthalle de Hambourg, organisé, dans les années 1970, une suite d'expositions marquantes sur la peinture en Europe autour de 1800, centrée sur quelques grandes figures (Friedrich, Füssli, Blake, Sergel, Turner, Runge, Flaxman, Goya), où la France est présente, certes, mais en rapport avec elles et non comme au centre. Les contraintes économiques l'avaient peut être empêché d'organiser une exposition analogue sur David ou sur Ingres, il n'empêche qu'avec cette série la peinture française n'apparaissait plus que comme un élément parmi d'autres d'un mouvement plus général de renouvellement où elle n'occupait pas forcément la première place. Une époque en rupture, où la plus large place est faite à David et à Ingres, mais aussi à Géricault et Delacroix aussi bien qu'à l'art de la Révolution, marque de ce point de vue un nouvel équilibre, sans toutefois minorer l'importance des artistes non-français, associés dans la naissance du mouvement romantique. Encore s'agit-il là d'approches relativement traditionnelles: beaucoup plus novateurs en effet en termes d'interprétation sont David Irwin, dans sa synthèse sur le néo-classicisme ${ }^{14}$, et Matthew Craske, dans la sienne sur l'art européen entre 1700 et $1730{ }^{15}$. Courts, ramassés, comme c'est la loi dans le genre étroit de livres orientés tout aussi bien vers 
le "grand public » que vers celui, plus restreint, des étudiants et des chercheurs, ils font en effet tous les deux une large place aux autres arts que la peinture, dans une approche où l'évolution stylistique compte autant que celle des modes de production, de réception et en dernier ressort de consommation de l'œuvre d'art, et où, assez paradoxalement, la politique ne tient pas toujours la première place au profit du champ économique et social. La longue ou la moyenne durée l'emporte ainsi sur le court terme, et l'art français de la Révolution ou de l'Empire garde d'une certaine manière sa spécificité, tout en restant un point d'ancrage fort de l'analyse ${ }^{16}$.

Au-delà de ces panoramas d'ensemble, c'est dans le détail même de la recherche qu'apparaissent, dans un phénomène cumulatif, les problématiques ouvertes autour du Bicentenaire et constamment enrichies depuis. On retiendra tout d'abord combien a progressé l'histoire des institutions artistiques et plus généralement du contexte intellectuel dans lequel elles s'inscrivent. Édouard Pommier avait, dans son édition des Lettres à Miranda de Quatremère de Quincy, ouvert la voie à une réflexion sur les doctrines et les idées sur les arts pendant la Révolution ${ }^{17}$, réflexion poursuivie dans sa synthèse, L'Art de la liberté ${ }^{18}$, et étendue ensuite dans deux directions plus précises, la figure emblématique et capitale de Winckelmann, considéré comme un des théoriciens les plus influents, si ce n'est le plus influent de l'époque, d'une part ${ }^{19}$, et, de l'autre, le problème $\mathrm{du}$ Musée ${ }^{20}$. Winckelmann, pendant longtemps assez négligé par l'historiographie française, a bénéficié, ces dernières années, d'un regain d'intérêt bienvenu, manifesté en particulier par l'édition ou la réédition de ses textes ${ }^{21}$. Mais c'est la question du musée qui a été profondément renouvelée, le bicentenaire de l'ouverture du Louvre, en 1993, correspondant à la rénovation aboutissant, peu après, à l'ouverture du «Grand Louvre ». Andrew MacClellan avait ouvert la voie ${ }^{22}$, Dominique Poulot s'imposant néanmoins comme le spécialiste de la question, élargie à celle de la naissance de la notion de patrimoine, au-delà même de la Révolution ${ }^{23}$. Le Louvre, dont la vraie naissance remonte à l'Ancien Régime, fut, on le sait, profondément modifié dans son essence dès 1795-1796 par les confiscations et spoliations opérées en Europe à la suite des victoires remportées par les armées françaises, et ce, jusqu'au Musée Napoléon, les défaites de 1814 et surtout 1815 aboutissant à des restitutions ellesmêmes constitutives de nombreuses collections publiques dans les pays d'origine. Ce double processus est désormais beaucoup mieux connu, en particulier grâce à l'exposition Vivant Denon organisée au Louvre en 1996. Denon fut au cœur du Musée Napoléon, comme d'ailleurs de la vie des arts sous l'Empire. L'exposition permit d'aborder toutes les facettes non seulement de sa riche personnalité, entre l'Ancien Régime et la Restauration, et par là toute une époque, mais aussi tous les domaines où se déploya son intense activité, c'est-à-dire pratiquement tous les arts ${ }^{24}$. Un remarquable colloque associé à l'exposition ${ }^{25}$ permet d'approfondir les divers points abordés dans le catalogue, complété par l'exceptionnelle publication de la correspondance officielle de Denon, même incomplète (tous les fonds d'archives n'ont pas été systématiquement dépouillés et confrontés) ${ }^{26}$. On peut regretter que le bicentenaire du décret pris par Chaptal en août 1801, créant véritablement les musées de province sur le modèle du Louvre, n'ait pas été l'occasion d'un travail analogue ${ }^{27}$. En revanche, la question, délicate, des spoliations et confiscations opérées en Europe commence à être beaucoup mieux étudiée, en particulier le cas allemand, grâce aux travaux de Bénédicte Savoy ${ }^{28}$. Enfin, la continuité des institutions muséographiques nées de la Révolution et de l'Empire avec celles de la Restauration, tant dans les doctrines que dans les personnels administratifs, peu modifiés en dehors des 
principaux responsables, a été bien mise en évidence par Marie-Claude Chaudonneret 29. Au total, c'est un nouveau chapitre de l'histoire institutionnelle des arts qui s'est ainsi peu à peu mis en place. Il existe bien encore des manques: ainsi le bouleversement entraîné par la suppression de l'Académie royale de peinture et de sculpture en 1793 et la mise en place subséquente d'un nouveau système d'enseignement, en particulier sous l'Empire (mais l'École des Beaux-Arts fut créée par la Restauration), ainsi que l'évolution du Salon et des expositions publiques, à Paris et en province, entre 1789 et 1815, sont loin d'avoir fait l'objet d'études aussi approfondies ${ }^{30}$. Et les publications de source, en particulier celles des correspondances officielles, se font encore attendre, alors qu'elles sont (depuis plus de cent ans !) disponibles pour l'ensemble du dix-huitième siècle ${ }^{31}$. Mais au total ce champ d'investigation, malgré ses manques et ses vides, a néanmoins été considérablement défriché et travaillé depuis une quinzaine d'années.

6 On aura remarqué qu'en abordant l'histoire institutionnelle, on a dû lier l'Ancien Régime, la Révolution, l'Empire et d'une certaine manière la Restauration. Cette extension, qui apparaît nécessaire à partir du moment où l'on cherche à inscrire dans un contexte plus large les créations artistiques de l'époque révolutionnaire, fait en même temps ressortir les continuités et les ruptures introduites par l'époque ellemême. L'approche monographique, sur laquelle on va revenir amplement à propos de la peinture et de la sculpture, favorise naturellement cette extension. Mais cette dernière est aussi de plus en plus soulignée par les historiens d'art de la période. Elle commence par la mise en perspective côte à côte, de la peinture, de la sculpture et des arts décoratifs, comme cela vient d'être fait, à partir des collections du Louvre, pour l'Empire ${ }^{32}$. Mais il est encore plus révélateur que vienne de paraître un remarquable recueil d'études sur la création artistique sous l'Empire, qui va de la littérature à la peinture en passant par la musique et la sculpture ${ }^{33}$, sur le même modèle et sous une direction identique, celle de Jean-Claude Bonnet, qu'un volume analogue publié, pour les années révolutionnaires, à l'occasion du Bicentenaire ${ }^{34}$. Mais alors qu'en 1989 l'accent était encore très littéraire, l'équipe réunie étant dans son écrasante majorité constituée d'historiens des lettres, on sent, dans celui consacré aux arts sous Napoléon, une démarche véritablement pluridisciplinaire, particulièrement bienvenue, sensible dans un choix d'intervenants beaucoup plus divers. Cette double extension, extension chronologique comme du domaine d'investigation, est porteuse de renouvellement et c'est, sans aucun doute, une direction d'avenir. C'est dans cette perspective que s'inscrivait, ainsi, l'exposition L'invention du sentiment au musée de la Musique en 2002, cherchant à lier dans un même mouvement peinture, sculpture, arts graphiques, littérature et musique ${ }^{35}$.

7 Ce renouvellement a néanmoins été tout aussi sensible dans le détail de la recherche, concentrée cette fois sur des champs précis, à commencer par celui de la création picturale. Une personnalité, écrasante, celle de Jacques-Louis David, domine la période. L'exposition du Louvre en 1989, le colloque qui lui avait été associé avaient en effet clairement montré l'existence, sur un même objet, de plusieurs courants méthodologiques : une approche, classique, partant de l'étude de l'œuvre, fondée aussi sur d'abondantes recherches documentaires, en particulier dans les dépôts d'archives, appuyait l'exposition, une position revendiquée et assumée par le principal auteur du catalogue, Antoine Schnapper ${ }^{36}$. Beaucoup plus ouvert, méthodologiquement, se voulait en revanche le colloque, organisé par Régis Michel, conservateur au Département des Arts graphiques du Musée du Louvre ${ }^{37}$. Le titre même qu'il avait 
retenu, David contre David, démontrait s'il en était besoin la volonté d'élargir le débat à toutes les formes de l'histoire de l'art, même si celles-ci ne sont justement pas acceptées par tous les historiens d'art. Véritablement international, faisant s'affronter ou à tout le moins se rencontrer toutes les interprétations possibles de la création artistique, David contre David reste une des meilleures démonstrations de la richesse que peut offrir la diversité des points de vue à partir du moment où tous sont équitablement représentés (le colloque organisé quelques années plus tard, toujours au Louvre, par le même Régis Michel, à l'occasion de l'exposition Géricault souffrant justement du resserrement de la perspective aux seuls points de vue « modernistes » ou « interprétatifs»). La distinction entre une histoire de l'art factuelle, fondée sur le dépouillement des sources et des documents, vite taxée de "positiviste », et une autre privilégiant l'interprétation des œuvres, sans craindre de leur imposer un cadre préétabli, recèle, il est vrai, une part d'arbitraire et d'artificiel. Elle ne recoupe pas forcément, d'ailleurs, la séparation, encore très présente en France si pas à l'étranger, entre deux professions, celle de conservateur et celle d'enseignant ou de chercheur, c'est-à-dire entre deux lieux principaux d'exercice, le musée et l'université. Elle dénote simplement deux orientations différentes dans ce que privilégie l'analyse, si tant est qu'il faille toujours choisir entre l'histoire et l'esthétique, l'histoire, par ailleurs, étant toujours une interprétation, comme le montrent les livres, très discutés chez les historiens d'art, de Thomas Crow, posant un regard neuf sur les rapports entre peinture et politique. Symbolique des effets entraînés par l'exposition David, Painting and Public Life in Eighteenth Century Paris, publié en 1985, n'a ainsi été traduit en français qu'en $200{ }^{38}$, alors que celle d'Emulation. Making Artists for Revolutionary France, consacré aux relations de David et ses élèves, n'a pris que deux ans ${ }^{39}$. La question politique est à chaque fois au centre de la problématique, mais intimement liée à l'analyse des œuvres. David était-il révolutionnaire, voire républicain, avant 1789 ? Comment ses élèves, étroitement associés à sa pratique picturale par les règles et les traditions de la formation académique, surent-ils s'émanciper de sa tutelle et prendre leur indépendance? Tant par son écriture que sa manière très particulière de bâtir un discours historique très problématisé sans pour autant rejeter toute analyse d'ordre stylistique ou esthétique, et donc en y intégrant étroitement les œuvres, Thomas Crow est sans conteste un point d'ancrage dans les débats encore ouverts sur la peinture française des années 1780 aux années 1820. Si un Todd Porterfield se situe dans la même ligne en analysant la représentation, dans la peinture française, des conflits menés au Proche-Orient et en Afrique du Nord, donnant ainsi aux débuts de la peinture orientaliste en France sa nécessaire coloration politique ${ }^{40}$, beaucoup plus discutables car beaucoup plus systématiques dans la projection et l'application au passé de problématiques contemporaines - sont, sur un sujet analogue, Extremities de Darcy Grimaldo Grigsby ${ }^{41}$, les analyses d'Abigail Salomon-Godeau sur "l'éroticisation » du corps masculin dans la peinture néo-classique française ${ }^{42}$, ou celles d'Eva LajerBurckardt sur la carrière de David après $1795^{43}$. Séduisants sans aucun doute d'un strict point de vue intellectuel, ces divers ouvrages ni disqualifient pas pour autant des études plus traditionnelles, mais tout aussi stimulantes finalement, comme l'étude de Tony Halliday sur le portrait ${ }^{44}$, les deux catalogues tout récemment consacrés par Philippe Bordes au "dernier David » ${ }^{45}$, celui de Sylvain Laveissière sur Le Sacre ${ }^{46}$ ou, toujours concernant David, le catalogue raisonné de son œuvre dessiné, source désormais incontournable ${ }^{47}$. 
8 Les principaux peintres français, contemporains de David, n'ont pas, jusqu'à présent, bénéficié de recherches aussi approfondies. On ne dispose en effet d'aucune monographie ou étude d'ensemble sur Gros, Guérin, Gérard ou Girodet. Aucune exposition ni ouvrage de synthèse (à l'exception de celui de Thomas Crow dont il vient d'être question - encore celui-ci ne vise-t-il pas à l'exhaustivité) ne leur a été consacré (la seule exposition d'envergure organisée en France ou à l'étranger depuis De David à Delacroix. Les Années romantiques, commençant justement après l'Empire [48]. Paradoxalement, c'est celui que David détestait, Prud'hon, qui a été le seul véritablement étudié ces dernières années ${ }^{49}$. Gros n'a été ainsi abordé que sous l'angle étroit de la seule peinture d'histoire, à partir de Napoléon sur le champ de bataille d'Eylau ${ }^{50}$, mais il fait exception (à noter, dans la même perspective, le volume très descriptif publié à partir des collections du Château de Versailles [51]. La situation devrait néanmoins rapidement évoluer. De nombreuses thèses sont en effet achevées, en attente de publication, ou en cours. Plusieurs importantes publications de documents, celle des inventaires après décès de Gros et de Girodet ${ }^{52}$, ou encore de Guérin ${ }^{53}$, témoignent d'une intense activité de recherche. La rétrospective Girodet, prévue pour l'automne $2005 \mathrm{au}$ Louvre et circulant ensuite au Canada et aux États-Unis, dont la préparation aura elle aussi été l'occasion de remettre au jour des sources capitales (notamment la correspondance de l'artiste, publiée très incomplètement juste après sa mort et jamais rééditée depuis), devrait sans aucun doute marquer un regain d'intérêt pour toute une génération de peintres, entre l'Ancien Régime et la Restauration.

9 En dehors de la grande peinture d'histoire, deux genres particuliers ont plus particulièrement retenu l'attention des historiens d'art ces dernières années. Le premier, le style «troubadour ", avait déjà été mis en valeur par les travaux de François Pupil et de Marie-Claude Chaudonneret ${ }^{54}$. Achetés par les musées (le Louvre à Paris, le musée des Beaux-Arts de Lyon, le musée de Grenoble et le musée de Brou à Bourg-enBresse), les peintres "troubadour ", spécialisés dans la représentation d'une histoire anecdotique, souvent sentimentale, située principalement au Moyen Âge ou à la Renaissance, et dans un «faire» minutieux et illusionniste marqué par l'exemple des peintres hollandais, concrétisent sous l'Empire, avec un très grand succès, des tendances nées en fait sous Louis XVI avec la série des tableaux illustrant l'histoire de France commandés par le directeur des Bâtiments du Roi, d'Angiviller. Ce courant, qui s'agrège au renouveau d'intérêt pour le Gothique caractéristique du Romantisme ${ }^{55}$, est aussi représentatif d'un mécénat particulier, aristocratique et féminin, dont les principales figures furent Joséphine et la Reine Hortense. L'étude de la collection de peinture de Joséphine ${ }^{56}$ a bien mis cet aspect en valeur, et constitue, en même temps, une étape dans l'analyse, sous l'angle du goût et des collections, de la période révolutionnaire et impériale, chantier de grande envergure et domaine d'avenir comme viennent de le montrer deux colloques successifs organisés par l'Institut National d'Histoire de l'Art ${ }^{57}$. Deuxième grand champ d'investigation dans les genres « mineurs » de la peinture, la peinture de paysage. On sait que celle-ci est sans conteste un des genres les plus populaires, hier comme aujourd'hui, de la peinture française du XIX siècle. C'est à partir d'une de ses figures majeures, Corot, que les recherches se sont portées sur les années 1780-1820, jusque là encore peu étudiées. Trois voies principales peuvent être retenues ici. La première a consisté à remonter si l'on peut dire la généalogie artistique de Corot, d'abord Achille-Etna Michallon, qui fut brièvement son professeur ${ }^{58}$, puis Pierre-Henri de Valenciennes, qui fut, à l'École des Beaux-Arts, le professeur de perspective de Michallon. Valenciennes apparaît en fait 
comme une des figures majeures du paysage néo-classique. La présentation de ses esquisses sur le motif, conservées en majeure partie au Louvre, en $1976{ }^{59}$, avait déjà constitué une étape capitale dans la reconstitution de l'évolution de la peinture de paysage à la charnière des dix-huitième et dix-neuvième siècles. Théoricien (il publia en 1800 des Éléments de perspective pratique qui sont en fait un véritable traité de la peinture de paysage), enseignant, Valenciennes exerça sans conteste une très grande influence. Plusieurs expositions ont ainsi remis son œuvre en perspective ${ }^{60}$. On ne peut néanmoins comprendre ce courant en l'isolant du reste de la peinture de paysage européenne, et l'Italie, avec Rome, joue ici un rôle essentiel. C'est ce qu'a parfaitement montré Anna Ottani Cavina avec Les Paysages de la Raison, montrant tout à la fois le creuset artistique international de la Rome des années 1780 , le rôle joué par la pratique du dessin autour de David et de l'Académie de France, le style insistant sur la ligne et les contrastes qui en résultèrent ainsi que l'évolution induite dans la peinture de paysage proprement dite (le livre s'attachant d'abord à la représentation de la ville) ${ }^{61}$. Anna Ottani Cavina, a ensuite étendu ses recherches à l'ensemble de la peinture de paysage de la fin du dix-huitième et du début du dix-neuvième siècles dans une exposition mémorable, Paysages d'Italie ${ }^{62}$, dans un mouvement chronologique inverse à celui suivi quelques années auparavant par un autre grand spécialiste de la peinture française, Philip Conisbee, cherchant à remonter aux sources de Corot ${ }^{63}$.

10 Beaucoup moins spectaculaires, à première vue, sont les développements de la recherche dans les deux derniers domaines que j'aborderai ici plus brièvement, la sculpture et les arts décoratifs. Domaines très spécialisés, moins favorisés par les expositions que la peinture, ils ne sont pourtant pas délaissés et les progrès, moins apparents peut-être que pour la peinture, n'en sont pas moins réels. Ils passent d'abord, plus encore que pour la peinture, par la connaissance précise des œuvres, leur identification aux sources d'archives, qui permet ensuite la restitution de leur réception et de leur circulation.

11 Si la sculpture de la Révolution et de l'Empire a bénéficié de la réévaluation, voire de la réhabilitation de la sculpture française du dix-neuvième siècle au milieu des années 1980, marquée par la grande exposition du Grand Palais dont le catalogue est encore aujourd'hui un livre essentiel ${ }^{64}$, tant pour les faits rassemblés que leur mise en perspective et leur problématisation, elle n'a pas acquis le degré de popularité de celle de la seconde moitié du siècle, mise en valeur par l'ouverture du musée d'Orsay. Les années 1789-1815 ont certes été favorisées, dans ce domaine, par l'ouverture du "Grand Louvre ", qui a mis sous les yeux du public nombre de sculptures autrefois dans les réserves, et le mouvement est analogue dans de nombreux musées en région, qui ont « ressorti » leurs ensembles sculptés au fur et à mesure des rénovations (Amiens, Grenoble, Lille, Lyon, Nantes pour les plus importants - seule la collection de Grenoble [ $\left.{ }^{65}\right]$ et, depuis peu, celle du musée Granet à Aix-en-Provence [ $\left.{ }^{66}\right]$ ont fait l'objet de catalogues complets). Mais elles restent le premier chapitre du dix-neuvième siècle, non une période étudiée en tant que telle, ainsi , par exemple dans l'ouvrage fondamental d'Antoinette Le Normand-Romain sur la sculpture funéraire ${ }^{67}$, dans celui de June Hargrove sur la représentation des "grands hommes " ${ }^{68}$ ou des études plus ponctuelles sur les monuments parisiens ${ }^{69}$. Est-il possible d'envisager une rétrospective de la sculpture française néo-classique? Les problèmes de coût et la difficulté de déplacer les œuvres la rendent peu envisageable. Exposer maquettes, esquisses et réductions pose moins de problèmes et l'on dispose ainsi aujourd'hui, par ce moyen, de deux excellentes vues d'ensemble de la période, envisagées soit sous un angle 
strictement français, soit plus européen, toutes deux trouvant leur origine au Louvre ${ }^{70}$. C'est en effet au Louvre que se situe l'épicentre de la recherche sur la sculpture française de cette époque, la collection, probablement la plus riche et la plus représentative au monde en la matière, motivant l'organisation régulière, depuis une dizaine d'années, d'expositions monographiques souvent accompagnées de colloque, qui renouvellent lentement, mais en profondeur, notre connaissance du sujet. À Clodion ${ }^{71}$ ont ainsi succédé Pajou ${ }^{72}$, puis Houdon (à Versailles) ${ }^{73}$, pendant que tout récemment Boizot ${ }^{74}$ et Julien ${ }^{75}$ étaient présentés en dehors de Paris. Si Deseine, Chaudet ${ }^{76}$ ou Chinard ${ }^{77}$ manquent encore à l'appel, la connaissance de la plupart des grands noms de la sculpture française autour de 1800 est désormais bien établie sur des études solides et récentes, complétée par des recherches plus ponctuelles d'ordre cette fois thématique, ainsi les portraits de Napoléon (mais on attend encore des monographies poussées, au-delà de la seule vulgarisation, sur des monuments aussi emblématiques que la Colonne Vendôme ou l'Arc de triomphe du Carrousel) ${ }^{78}$. On ne saurait enfin aborder la sculpture de la période révolutionnaire et impériale sans mentionner le nom de Canova, dont la carrière fut en partie orientée par les événements de France, sans compter qu'une part importante s'y déroula. L'ouverture des collections russes, en l'espèce celles de l'Ermitage, qui par l'héritage du Prince Eugène recèle une partie des sculptures de Canova rassemblées par Joséphine, l'activité de la Gypsothèque de Possagno qui conserve le fond d'atelier de l'artiste, et plus généralement le renouveau d'intérêt pour le néo-classicisme ont ajouté plusieurs ouvrages à la considérable bibliographie canovienne. Retenons ici les deux catalogues rétrospectifs de $1992{ }^{79}$ et $2003{ }^{80}$, ainsi que l'étude de Christopher Johns sur les relations de Canova et de ses commanditaires ${ }^{81}$, en y ajoutant, tant l'œuvre (qui fut achetée par Murat en Italie) illustre, en France, l'art de Canova, la monographie d'Isabelle Leroy-Jay-Lemaistre sur Psyché ranimée par le baiser de l'Amour ${ }^{82}$.

12 Les recherches dans le domaine des arts décoratifs, très dispersées dans les revues spécialisées, n'offrent encore que des synthèses limitées : la remarquable exposition du musée Carnavalet, $\mathrm{Au}$ temps des merveilleuses, aborde certes ce domaine, mais en concurrence avec les autres formes d'expression artistiques et si le catalogue est passionnant (encore que toutes les œuvres exposées n'y soient pas reproduites, ce qui est d'autant plus frustrant qu'il s'agit là de la première exposition sur le sujet), il ne constitue, finalement, qu'une première mise en place ${ }^{83}$. De même l'exposition du Musée des tissus et arts décoratifs de Lyon sur Lyon et Napoléon, quoique fort intéressante dans son ambition d'aborder tous les aspects économiques, industriels et sociaux liés à la production artistique, reste relativement circonscrite de par son ancrage volontairement régional ${ }^{84}$. La seule exposiiton d'ensemble ayant abordé de front un des aspects stylistiques majeurs du style Empire, Egyptomania n'était pas spécifiquement consacrée aux arts décoratifs ${ }^{85}$, de même que D'après l'antique, qui présentait, pour la période qui nous occupe, un développement passionnant sur un type particulier de meuble, l'athénienne ${ }^{86}$. On espère, pour la Révolution et l'Empire, voire le règne de Louis XVI qui en est ici inséparable, une exposition aussi complète et novatrice que fut Un âge d'or des arts décoratifs, consacré certes à la Restauration et à la Monarchie de Juillet, mais qui offrait des éléments intéressants sur la période antérieure ${ }^{87}$. Ici encore, la monographie est un nécessaire préalable : monographies d'artistes ${ }^{88}$ ou d'ensembles mobiliers ${ }^{89}$, mais aussi monographies de domaines comme Malmaison ${ }^{90}$, Trianon ${ }^{91} \mathrm{ou}$ Fontainebleau ${ }^{92}$, monographies de genre a priori plus arides comme les soieries ${ }^{93}$, les tissus ${ }^{94}$, le bronze doré ${ }^{95}$, la porcelaine ${ }^{96}$, l'orfèvrerie ${ }^{97}$ ou même les tapis ${ }^{98}$, mais 
révélatrices des évolutions des couleurs et de l'ornement. C'est le parti-pris du très séduisant ouvrage de Bernard Chevallier sur le style Empire, les photographies, d'une exceptionnelle qualité, ne révélant que rarement la totalité d'un meuble ou d'un ensemble, préférant le détail stylistique significatif au tout ${ }^{99}$. Le bicentenaire de la proclamation de l'Empire n'a pas donné lieu à des manifestations d'envergure : outre celle du musée de Lyon notée plus haut, l'exposition des œuvres rassemblées par la Fondation Napoléon ${ }^{100}$, ainsi qu'une autre sur Napoléon et Versailles, qui remet au premier plan les aménagements projetés au Château lui-même, connus par un album d'aquarelles de Percier et Fontaine, et les réalisations effectives au Grand Trianon ${ }^{101}$. Outre une exposition majeure sur les arts décoratifs entre 1789 et 1815, seule capable de faire synthétiquement un bilan des recherches actuelles, mais qui ne semble pas à l'ordre du jour, on peut espérer la poursuite des publications des intérieurs impériaux conservés et désormais à peu près tous restaurés (Malmaison, Fontainebleau, Compiègne, Trianon). Le projet de recherches menés au sein du Centre allemand d'histoire de l'art à Paris qui accompagne la restauration des intérieurs de l'Hôtel Beauharnais à Paris (actuelle ambassade d'Allemagne) apportera sans nul doute également beaucoup.

13 Je terminerai cette introduction historiographique sur une note plus personnelle. Ayant consacré une partie de mes propres recherches à l'estampe, d'une part, et à l'art britannique, de l'autre, je suis toujours frappé, d'une part, par le peu d'intérêt suscité par la première, et, de l'autre, par une tendance à mon avis malheureuse des historiens de l'art français à s'aventurer hors de nos frontières. Il est vrai que Pascal Dupuy a fait l'un et l'autre à l'occasion de sa thèse, mais le cas reste exceptionnel ${ }^{102}$. Je souhaiterais, pour conclure, que la gravure (et la lithographie qui naît justement en 1798, au cœur de la période ici abordée), soit davantage étudiée à l'avenir, et que l'on n'hésite pas, non plus à risquer les comparaisons avec les artistes étrangers. Un des grands acquis de la rétrospective consacrée il y a deux ans à William Blake à Londres, et des publications qui l'ont accompagnée ${ }^{103}$, a été justement de montrer comment sa création, dans les années révolutionnaires, a véritablement accompagné les nouvelles venues de France et la réaction qu'elles suscitèrent en Grande-Bretagne. Il y a là un enseignement à méditer.

\section{NOTES}

1.Ainsi Annie JouRdAn, Les Monuments de la Révolution 1770-1804 : une histoire de représentation, Paris/Genève, 1997 ; Napoléon, héros, imperator, mécène, Paris, 1998; Mythes et légendes de Napoléon : un destin d'exception, entre rêve et réalité, Toulouse, 2004.

2.Robert RosenBlum, Transformations in Late Eighteenth Century Art, Princeton, 1967, trad.

fr. L'art au XVIIIe siècle. Transformations et mutations, Saint-Pierre de Salerne, 1989.

3.The Age of Neo-Classicism, Arts Council of Great Britain, 1972 (l'exposition avait lieu à la Royal Academy et au Victoria \& Albert Museum).

4.De David à Delacroix. La peinture française de 1774 à 1830, Paris, Galeries nationales du Grand Palais, 1974. 
5.Le Néo-Classicisme français. Dessins des musées de province, Paris, Galeries nationales du Grand Palais, Paris, 1974, exposition précédée, deux ans auparavant, de Dessins français de 1750 à 1825. Le Néo-Classicisme, Paris, Musée du Louvre, 1972.

6.Philippe BORDES et Régis Michel (dir.), Aux Armes et aux Arts! Les Arts de la Révolution, 1789-1799, Paris, 1988.

7.La Révolution française et l'Europe, 1789-1799, Paris, Galeries nationales du Grand Palais, 1989.

8.Philippe Bordes et Alain CHEVALIER, Catalogue des Peintures, Sculptures et Dessins, Vizille, Musée de la Révolution française, 1996.

9.Lucius Junius Brutus. L'Antiquité et la Révolution française, Vizille, Musée de la Révolution française, 1996.

10.Le Portrait de Lally-Tollendal : un chef-d'œuvre du musée, Vizille, Musée de la Révolution française, 2005.

11.La Révolution par la gravure : les "Tableaux historiques de la Révolution française ", une entreprise éditoriale d'information et sa diffusion en Europe (1791-1817), Vizille, Musée de la Révolution française, 2002.

12.William VAUGHAN, L'Art du XIXe siècle, 1780-1850, Paris, 1989 (coll. « Les grandes civilisations $»)$.

13. Werner HofmanN, Une époque en rupture, 1750-1830, Paris, 1995 (coll. « L'Univers des formes »).

14.David IRWIN, Neoclassicism, Londres, 1997 (coll. « Art and Ideas »).

15. Matthew CRASKE, Art in Europe 1700-1830, Oxford, 1997 (coll. « Oxford History of Art »). 16.Ainsi dans le stimulant recueil d'articles édité par Andrew HEMINGWAY et William VAUGHAN, Art in Bourgeois Society, 1790-1850, Cambridge, 1998.

17.Édouard POMMIER (éd.), Quatremère de Quincy : Lettres à Miranda sur le déplacement des monuments de l'art de l'Italie (1796), Paris, 1989.

18.Id., L'Art de la liberté. Doctrines et débats de la Révolution française, Paris, 1991.

19.Id., Winckelmann, inventeur de l'histoire de l'art, Paris, 2003.

20.Id. (dir.), Le Musée en Europe à la veille de l'ouverture du Louvre. Actes du colloque organisé par le service culturel du Musée du Louvre le 3, 4, 5 juin 1993 sous la direction scientifique d'Édouard Pommier, Paris, 1995.

21.Voir Édouard Pommier (éd.), Winckelmann : la naissance de l'histoire de l'art à l'époque des Lumières. Actes du cycle de conférences prononcées à l'Auditorium du Louvre du 11 décembre 1989 au 12 février 1991, Paris, 1991 ; Alex POTTs, Flesh and the ideal : Winckelmann and the origins of Art History, New haven et Londres, 1994 ; Jackie PIGAULT et Jean-Paul BARBE éd., Winckelmann et le retour à l'antique. Entretiens de la Garenne-Lemot, Actes du colloque des 9-12 juin 1994, Nantes, 1995 ; Elisabeth DÉculTOT, Johann Joachim Winckelmann : enquête sur la genèse de l'histoire de l'art, Paris, 2000. Une étude de Daniela Gallo est attendue prochainement sur le sujet.

22. Andrew McCLELLAN, Inventing the Louvre: Art, Politics, and the Origins of the modern Museum in Eighteenth Century Paris, Cambridge, 1994.

23.Dominique Poulot, Bibliographie de l'histoire des musées de France, Paris, 1994 ;

Surveiller et s'instruire : La Révolution française et l'intelligence de l'héritage historique, Oxford, 1996 ; Musée, nation, Patrimoine, 1789-1815, Paris, 1997 ; Patrimoine et musées :

l'institution de la culture, Paris, 2001 
24.Pierre Rosenberg et Marie-Anne Dupuy (dir.), Dominique Vivant Denon. L'œil de Napoléon, Paris, Musée du Louvre, 1999.

25.Daniela Gallo (dir.), Les vies de Dominique Vivant Denon. Actes du colloque organisé au musée du Louvre par le service culturel du 8 au 11 décembre 1999, Paris, 2001.

26.Marie-Anne DUPUY, Isabelle LE MASNE DE CHERMONT, Elaine wILlIAMSON éd., Vivant Denon, Directeur des musées sous le Consulat et l'Empire : correspondance, 1802-1815, Paris, 1999.

27. À noter néanmoins le catalogue de l'exposition commémorative du Musée des Beaux-Arts de Nancy, qui avait reconstitué les premiers accrochages et la collection d'origine : Le Premier musée de Nancy, de l'an II au Sacre de Napoléon, Nancy, Musée des Beaux-Arts, 2001-2002.

28. Bénédicte SAVOY, Patrimoine annexé. Les biens culturels saisis par la France en Allemagne autour de 1800, Paris, 2003. Le deuxième volume, établi avec la collaboration de Nicolas Labasque, étudie plus précisément l'exposition faite par Vivant Denon des œuvres saisies après les campagnes de Prusse et d'Autriche, en 1807-1809. Pour la discussion engendrée par ces confiscations, voir Bénédicte SAvoy (éd.) (trad. par Aurélie Duthoo), Johann Friederich Ferdinand Emperius, Témoignages sur les saisies d'art opérées en Allemagne par Vivant Denon, Paris, 1999. Pour le contexte des restitutions, voir Bénédicte SAVoY (éd.)(avec la collab. De Nicolas Labasque), Jean Henry. Journal d'un voyage à Paris en 1814, Paris, 2001.

29.Marie-Claude CHAUdONNERET, L'État et les artistes, de la Restauration à la Monarchie de Juillet, 1815-1833, Paris, 1999.

30.Le bicentenaire de l'installation de l'Académie de France à Rome à la villa Médicis a néanmoins donné lieu à une très importante exposition, qui concerne surtout le dixneuvième siècle dans son ensemble : Olivier BonfAIT (dir.), Maestà di Roma. Di Napoleone all'unità d'Italia. D'Ingres à Degas. Les artistes français à Rome, Rome, Académie de France, 2003. Un projet d'ensemble sur les Salons, coordonné par l'UMR André Chastel/ Université de Paris IV-Sorbonne, en liaison avec la B.N.F. et les musées du Louvre et d'Orsay, est en cours. Un colloque consacré aux Salons sous l'Empire, organisé à la Bibliothèque Marmottan, Boulogne-Billancourt, en 2003, est en cours de publication avec l'aide de la fondation Napoléon. La réédition systématique des catalogues de livrets des Salons et expositions du dix-neuvième siècle, actuellement entreprise par les éditions de l'Échelle de Jacob, à Paris et à Dijon, rend plus accessible une source précieuse, mais reprise en reprint telle quelle, sans aucune annotation.

31.Les premiers volumes de la nouvelle série de la Correspondance des directeurs de l'Académie de France à Rome, publiés sous l'égide de la Société de l'Histoire de l'Art français, étaient attendus pour le printemps 2005.

32.Sylvain LAVEISSIÈRE (dir.), Napoléon et le Louvre, Paris, 2004.

33.Jean-Claude BONNET (dir.), L'Empire des Muses. Napoléon, les arts et les lettres, Paris, 2004.

34.Id. (dir.), La Carmagnole des Muses. L'homme de lettres et l'artiste dans la Révolution, Paris, 1988.

35.Frédéric DASSAS, Dominique de FONT-RÉAULX et Barthélémy JOBERT (dir.), L'Invention du sentiment. Aux sources du Romantisme, Paris, Cité de la Musique, 2002, à compléter par Barthélémy JOBERT (dir.), De la rhétorique des passions à l'expression du sentiment. Actes du colloque des 14, 15 et 16 mai 2002, Paris, 2003. 
36.Antoine SCHNAPPER et Arlette SÉRULLAZ (dir.), Jacques-Louis David, 1748-1825, Paris, Musée du Louvre, 1989.

37.Régis Michel (dir.), David contre David. Actes du colloque organisé au musée du Louvre par le service culturel du 6 au 10 décembre 1989, Paris, 1993.

38.Thomas CROW, La Peinture et son public à Paris au XVIIIe siècle, Paris, 2000.

39.Id., L'Atelier de David. Émulation et Révolution, Paris, 1997.

40.Todd PORTERFIELD, The Allure of Empire. Art in the Service of French Imperialism, 1798-1836, Princeton, 1998.

41.Darcy Grimaldo GRIGSBY, Extremities. Painting Empire in Post-Revolutionary France, New Haven et Londres, 2002.

42.Abigail Solomon-GodeAu, Male Trouble. A Crisis in Representation, Londres, 1997.

43.Ewa LAJER-BURCARDT, Necklines. The Art of Jacques-Louis David after the Terror, New Haven et Londres, 1999.

44.Tony Halliday, Facing the Public. Portraiture in the Aftermath of the French Revolution, Manchester, 2000.

45.Philippe BoRDES, Portraiture in Paris around 1800 : Cooper Penrose by Jacques-Louis David, San Diego, Ca., Timken Museum of Art, 2004 ; Philippe BoRDES, Jacques-Louis David, Empire to Exile, New Haven et Londres, 2005 (exposition tenue au J.P. Getty Museum of Art).

46.Sylvain LAVEISSIÈRE, Le Sacre de Napoléon peint par David, Paris, 2004.

47.Pierre ROSENBERG et Louis-Antoine PRAT, Jacques-Louis David, 1748-1825 : Catalogue raisonné des dessins, Milan, 2002. Le catalogue de la collection du Département des arts graphiques du Musée du Louvre avait été établi par Arlette Sérullaz en 1991 (Inventaire général des dessins, Ecole française. Dessins de Jacques-Louis David 1748-1825, Paris, 1991). 48. Les Années romantiques. La peinture française de 1815 à 1850, Nantes, Musée des BeauxArts, 1995 (exposition reprise à Paris, Grand Palais, 1996). L'ouvrage reste néanmoins utile pour les dernières années des peintres ayant travaillé sous la Révolution et sous l'Empire.

49.Sylvain LAVEISSIÈRE, Prud'hon ou le rêve du bonheur, Paris, Galeries nationales du Grand Palais, 1997.

50.Christopher PRENDERGAST, Napoleon and History Painting. Antoine-Jean Gros's « La Bataille d'Eylau », Oxford, 1997. Doit paraître à l'automne 2005 une analyse « politique » de la carrière de Gros par David o'BRIEN, Antoine-Jean Gros. Painting and Propaganda under Napoléon Bonaparte, à la fois à Philadelphie et à Paris (Penn State University Press et Gallimard).

51.Claire CONSTANS, Bruno FOUCART et Yveline CANTAREL-BESSON, Napoléon. Images et histoire. Peintures du Château de Versailles, Paris, 2001.

52.Valérie BAJOU et Sidonie LEMEUX-FRAITOT (éd.), Inventaires après décès de Gros et de Girodet. Documents inédits, Paris, 2002.

53.Josette BOTTINEAU et Élisabeth FOUCART-WALTER (éd.), L'Inventaire après décès de PierreNarcisse Guérin, Archives de l'art français, nouvelle période, tome XXXVII (2005).

54.François PUPIL, Le Style troubadour ou la nostalgie du bon vieux temps, Nancy, 1985 ; Marie-Claude CHAUDONNERET, La peinture troubadour, deux artistes lyonnais : Pierre Révoil (1776-1842), Fleury Richard (1777-1852), Paris, 1980; Fleury Richard et Pierre Révoil. La peinture troubadour, Neuilly-sur Seine, 1980.

55.Guy STAIR SAINTY (dir.), Romance and Chivalry: History and Literature Reflected in Early Nineteenth-Century French Painting, Stair Sainty Mathiesen Gallery, New York, 1996. 
56.Alain Pougetoux, La collection de peintures de l'Impératrice Joséphine, Paris, 2003. Voir également le Petit Journal des Grandes Expositions consacré à l'exposition tenue en 2003 à La Malmaison, Joséphine et ses peintres.

57.Colloques organisés avec la collaboration du Getty Research Institute : Collections et marché de l'art en France 1789-1848, Paris, 4-6 décembre 2003 ; Redistributions : révolution, politique, guerre et déplacements de l'art, 1789 - 1848, Paris, 9-11 décembre 2004. La publication des actes est en cours.

58.Vincent Pomarède (dir.), Achille-Etna Michallon, Paris, Musée du Louvre, 1994.

59.Geneviève LACAMBRE, Les Peintures de paysage de Pierre-Henri de Valenciennes, 1750-1819, Paris, Musée du Louvre, 1976.

60.On retiendra essentiellement Bruno MANTURA et Geneviève LACAMBRE (dir.), PierreHenri de Valenciennes 1750-1819, Spolète, Palazzo Racani Arroni, 1996; et « La nature l'avait créé peintre » : Pierre-Henri de Valenciennes, 1750-1819, Toulouse, Musée Paul Dupuy, 2003. Voir également Simone ScHUlTZE, Pierre-Henri de Valenciennes und seine Schule : Paysage historique" und der Wandel in der Naturauffassung am Anfang des 19. Jahrhunderts, Francfort, 1995.

61.Anna OTTANI CAVINA, I paesaggi della ragione: la città neoclassica da David a Humbert de Superville, Turin, 1994, traduction française Les Paysages de la Raison. La ville néo-classique de David à Humbert de Superville, Arles, 2005.

62.Id. (dir.), Paysages d'Italie. Les peintres du plein-air (1780-1830), Paris, Galeries nationales du Grand Palais, 2001.

63.Philip CONISBEE (dir.), avec Sarah FAUNCE, Jeremy STRICK et Peter GALASSI, In the Light of Italy. Corot and Early Open-Air Painting, Washington, National Gallery of Art, 1996.

64.Anne PINGEOT (dir.), La Sculpture française au XIXe siècle, Paris, Galeries nationales du Grand Palais, 1986.

65.Catherine CHEVILLOT (dir.), La collection du musée de Grenoble. Peintures et sculptures du XIXe siècle, Paris, 1995. À noter que les peintures de la Révolution et de l'Empire figurent dans un autre volume, Gilles CHOMER (dir.), La collection du musée de Grenoble. Peintures françaises avant 1815, Paris, 2000. Le musée de Grenoble est le seul musée français à avoir publié intégralement le catalogue de ses collections. 66.Alexandre MARAL, Sculptures : la galerie du Musée Granet, Aix-en-Provence et Paris, 2003.

67.Antoinette LE NORMAND-ROMAIN, Mémoire de marbre. La sculpture funéraire en France, 1804-1914, Paris, 1995.

68.June HARgrove, Les Statues de Paris. La représentation des grands hommes sur les places et dans les rues de Paris, Paris, 1989. À noter la parution, en 2004, du CD-ROM édité conjointement par le musée d'Orsay et l'I.N.H.A. À nos grands hommes. La sulpture publique en France jusqu'à la Seconde Guerre mondiale, répertoriant plus de 5000 monuments grâce à une collection d'environ 20000 cartes postales rassemblées par la collectionneuse France Debuisson, et qui couvre donc la période révolutionnaire et impériale.

69.Dominique MASSOUNIE, Pauline PRÉVOST-MARCILHACY et Daniel RABREAU (dir.), Paris et ses fontaines, de la Renaissance à nos jours, Paris, 1995 ; Geneviève BRESC-BAUTIER et Xavier DECTOT (dir.), avec l'aide de Ilham BEN BOUMEHDI, Art ou politique? Arcs, statues et colonnes de Paris, Paris, 1999 
70.Skulptur aus dem Louvre/Sculptures françaises néo-classiques 1760-1830, Paris, Musée du Louvre, 1990 ; James David DRAPER et Guilhem SCHERF (dir.), L'esprit créateur, de Pigalle à Canova. Terres cuites européennes, 1740-1840, Paris, Musée du Louvre, 2003.

71.Anne POULET et Guilhem SCHERF, Clodion, 1738-1814, Paris, Musée du Louvre, 1992 ; Guilhem SCHERF (dir.), Clodion et la sculpture française de la fin du XVIIIe siècle. Actes du colloque organisé par le service culturel du musée du Louvre les 20 et 21 mars 1992, Paris, 1993. 72.James DAVID DRAPER et Guilhem SCHERF, Pajou, sculpteur du Roi, 1730-1809, Paris, Musée du Louvre, 1997 ; Guilhem SCHERF (dir.), Pajou et ses contemporains. Actes du colloque organisé au Louvre par le service culturel les 7 et 8 novembre 1997, Paris, 2000.

73.Anne POULET, Guilhem SCHERF et Ulrich D. MATHIES, Houdon, sculpteur des Lumières, 1741-1828, Versailles, Musée national du Château, 2004 ; Voir aussi Guilhem SCHERF, Houdon : " Diane Chasseresse », Paris, 2000

74.Thérèse PICQUENARD, Guilhem SCHERF, Anne BILLON, Marie-Laure de ROCHEBRUNE et Christian BAULEZ, Louis-Simon Boizot, sculpteur du Roi et directeur de l'atelier de sculpture à la Manufacture de Sèvres, Versailles, Musée Lambinet, 2001.

75.Gilles GRANDJEAN et Guilhem SCHERF (dir.), Pierre Julien 1731-1804, Le Puy-en-Velay, Musée Crozatier, 2004.

76.Voir néanmoins Charles JANORAY, Chaudet's Belisarius : an Example of Virtue, New York, 2003. Une publication prochaine sur Chaudet est attendue.

77.Pour Chinard, on se reportera utilement au catalogue de l'exposition Les Muses de Messidor. Peintres et sculpteurs lyonnais de la Révolution et de l'Empire, Lyon, Musée des Beaux-Arts, 1989.

78.Gérard HUBERT et Guy LEDOUX-LEBARD, Napoléon. Portraits contemporains, bustes et statues, Paris, 1999.

79.Antonio Canova, Venise, Musée Correr et Possagno, Gypsothèque, 1992.

80.Canova, Bassagno del Grappa, Museo Civico et Possagno, Gypsothèque, 2003.

81. Christopher M. S. JoHNS, Antonio Canova and the Politics of Patronage in Revolutionary and Napoleonic Europe, Berkeley, Los Angeles et Londres, 1998.

82.Isabelle LEROY-JAY-LEMAISTRE, Canova : «Psyché ranimée par le baiser de l'Amour », Paris, 2003.

83.Jean-Marie BRUSON et Anne FORRAY-CARLIER (dir.), Au temps des Merveilleuses. La société parisienne sous le Directoire et le Consulat, Paris, Musée Carnavalet, 2005.

84.Lyon et Napoléon, Lyon, Musée des tissus et des arts décoratifs, 2005.

85.Jean-Marcel HUMBERT, Michael PANTAZzi et Christiane ZIEGLER (dir.), Egyptomania, Paris, Musée du Louvre, 1994, à compléter par Jean-Marcel HUMBERT (dir.), L'égyptomanie à l'épreuve de l'archéologie. Actes du colloque organisé par le service culturel du musée du Louvre les 8 et 9 avril 1994, Paris, 1996.

86.D’Après l'antique, Paris, Musée du Louvre, 2000.

87.Un âge d'or des arts décoratifs, 1814-1848, Paris, Galeries nationales du Grand Palais, 1992.

88. Hans OtTomeyer, Das Frühe CEuvre Charles Perciers (1780-1800), zu des Anfängen des Historismus in Frankreich, Munich, 1981 (auquel il faut ajouter, bien qu'il s'agisse d'une édition de texte qui concerne principalement l'architecture, l'édition du Journal 1799-1853 de Pierre François Léonard Fontaine, Paris, 1987) ; Denise LEDOUX-LEBARD, Le Mobilier français du XIXe siècle. Dictionnaire des ébénistes et des menuisiers, Paris, 1989 ; Ulrich LEBEN, Molitor, ébéniste de Louis XVI à Louis XVIII, Paris, 1992, à compléter par Bernard Molitor, 1755-1835, ébéniste parisien d'origine luxembourgeoise, Luxembourg, Villa 
Vauban, 1995 ; Marie-Noëlle de GRANDRY, Le mobilier français. Directoire, Consulat, Empire, Paris, 1996.

89.Un ameublement à la mode en 1802. Le mobilier du général Moreau, Fontainebleau, Musée national du Château, 1992.

90.Bernard CHEVALLIER, Malmaison, château et domaine des origines à 1904, Paris, 1989.

91.Denise LEDOUX-LEBARD, Le Grand Trianon. Meubles et objets d'art, Paris, 1975 ; Denise LEDOUX-LEBARD, Versailles. Le Petit Trianon. Le mobilier des inventaires de 1807, 1810 et 1839, Paris, 1989.

92.Jean-Pierre SAmoyault, Musée national du Château de Fontainebleau. Catalogue des collections de mobilier. Pendules et bronzes d'ameublement entrés sous le premier Empire, Paris, 1989 ; Jean-Pierre SAmoyault, Musée national du château de Fontainebleau. Catalogue des collections de mobilier. Meubles entrés sous le premier Empire, Paris, 2004 ; Bernard CHEVALLIER, Musée national du Château de Fontainebleau. Catalogue des collections de mobilier. Les Sèvres de Fontainebleau. Porcelaines, terres vernissées, émaux, vitraux (pièces entrées de 1804 à 1904), Paris, 1996 ; Jean-Pierre SAMOYAULT et Colombe SAMOYAULT-VERLET, Château de Fontainebleau. Musée Napoléon Ier. Napoléon et la famille impériale, Paris, 1986 ; Yves CARLIER, Vincent DROGUET, Amaury LEFÉBURE et Danièle VÉRON-DENISE, Napoléon à Fontainebleau, Paris, 2003.

93.Jean COURAL, Chantal GASTINEL-COURAL et Muriel MÛNTZ DE RAÏSSAC, Soieries empire. Mobilier national, Paris, 1980 ; Soies tissées, soies brodées chez l'impératrice Joséphine, Musée national des Châteaux de Malmaison et de Bois-Préau, 2003.

94.Mélanie RIFfel et Sophie ROUART, La Toile de Jouy, Paris, 2003.

95.Hans OTTOMEYER et Peter PRÖSCHEL, Vergoldete Bronzen, Munich, 1986.

96. Régine PLINVAL DE GUILLEBON, La porcelaine à Paris sous le Consulat et l'Empire, Paris.

97.Anne DION-TENENBAUM, L'Orfèvre de Napoléon, Martin-Guillaume Biennais, Paris, Musée du Louvre, 2003.

98.Tapis d'Empire. Maquettes de la collection Marmottan, Boulogne-Billancourt/Paris, Bibliothèque Marmottan, 2003.

99.Bernard CHEVALLIER, Style Empire. Les arts décoratifs de 1798 à 1815, Paris, 2000.

100.Trésors de la Fondation Napoléon. Dans l'intimité de la Cour impériale, Paris, Musée Jacquemart-André, 2004.

101.Jérémie BENOÎT, Napoléon et Versailles, Musée national du Château de Versailles, 2005.

102.Pascal DUPUY, L'Angleterre face à la Révolution. La représentation de la France et des Français à travers la caricature 1789-1802, thèse de doctorat soutenue à l'Université de Rouen, 1998 (en cours de publication).

103.Pour le point qui nous occupe ici, Robin HAMLYN et Michael PHILLIPS, William Blake, Londres, Tate Gallery, 2000 et Michael PHILliPs, William Blake. The Creation of the "Songs ». From Manuscript to Illuminated Printing, Londres, 2000. 


\section{AUTEUR}

\section{BARTHÉLÉMY JOBERT}

Professeur d'histoire de l'art moderne et contemporain (patrimoine) à l'Université de Paris IV-Sorbonne. UMR André Chastel, Institut National d'Histoire de l'Art, Galerie Colbert, 2 rue Vivienne 75002 Paris 